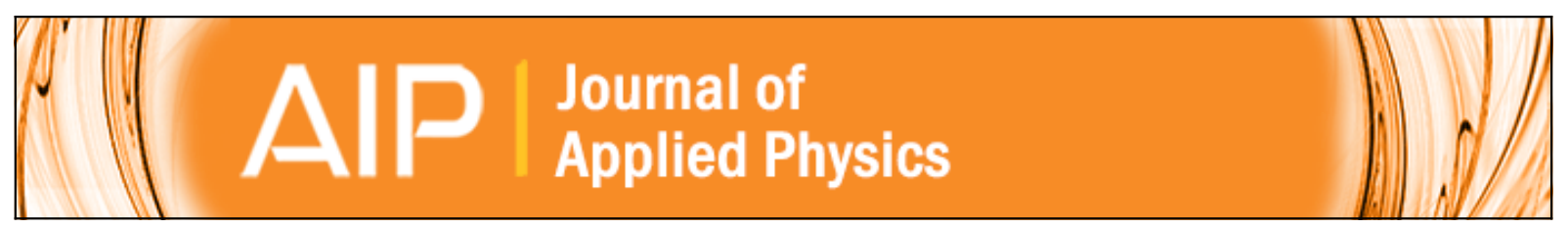

\title{
Numerical computation for a new way to reduce vibration and noise due to magnetostriction and magnetic forces of transformer cores
}

Lihua Zhu, Qingxin Yang, Rongge Yan, Yongjian Li, Xian Zhang, Weili Yan, and Jianguo Zhu

Citation: Journal of Applied Physics 113, 17A333 (2013); doi: 10.1063/1.4800077

View online: http://dx.doi.org/10.1063/1.4800077

View Table of Contents: http://scitation.aip.org/content/aip/journal/jap/113/17?ver=pdfcov

Published by the AIP Publishing

\section{Articles you may be interested in}

Magnetostrictive vibrations model of a three-phase transformer core and the contribution of the fifth harmonic in the grid voltage

J. Appl. Phys. 115, 17A316 (2014); 10.1063/1.4863931

Core loss behavior in high frequency high power transformers-I: Effect of core topology

J. Renewable Sustainable Energy 4, 033112 (2012); 10.1063/1.4727910

Effects of magnetomechanical vibrations and bending stresses on three-phase three-leg transformers with amorphous cores

J. Appl. Phys. 111, 07E730 (2012); 10.1063/1.3678459

Reducing audible noise for distribution transformer with HB1 amorphous core

J. Appl. Phys. 109, 07 A318 (2011); 10.1063/1.3553939

An assessment of alternative soft magnetic materials in rotary variable differential transformers

J. Appl. Phys. 81, 4295 (1997); 10.1063/1.364807

\section{High-Voltage Amplifiers}

-Voltage Range from $\pm 50 \mathrm{~V}$ to $\pm 60 \mathrm{kV}$

- Current to $25 \mathrm{~A}$

Electrostatic Voltmeters

- Contacting \& Non-contacting

- Sensitive to $1 \mathrm{mV}$

- Measure to 20kV
ENABLING RESEARCH AND

INNOVATION IN DIELECTRICS,

ELECTROSTATICS, MATERIALS, PLASMAS AND PIEZOS

TRek www.trekinc.com 


\title{
Numerical computation for a new way to reduce vibration and noise due to magnetostriction and magnetic forces of transformer cores
}

\author{
Lihua Zhu, ${ }^{1}$ Qingxin Yang, ${ }^{1,2}$ Rongge Yan, ${ }^{1, a)}$ Yongjian Li, ${ }^{1}$ Xian Zhang, ${ }^{2}$ Weili Yan, ${ }^{1}$ \\ and Jianguo $\mathrm{Zhu}^{3}$ \\ ${ }^{1}$ Province-Ministry Joint Key Laboratory of Electromagnetic Field and Electrical Apparatus Reliability, \\ Hebei University of Technology, Tianjin 300130, China \\ ${ }^{2}$ Municipal Key Laboratory of Advanced Technology of Electrical Engineering and Energy, \\ Tianjin Polytechnic University, Tianjin 300387, China \\ ${ }^{3}$ School of Electrical, Mechanical and Mechatronic Systems, University of Technology, Sydney, \\ NSW 2007, Australia
}

(Presented 16 January 2013; received 1 November 2012; accepted 14 January 2013; published online 8 April 2013)

\begin{abstract}
Magnetostriction (MS) caused by the global magnetization of limbs and yokes and magnetic forces are the undisputed causes of the vibration and noise in power transformer cores. This paper presents a novel way to reduce the vibration and noise, in which nanocrystalline soft magnetic composite (NSMC) material with high permeability is used to fill the step-lap joint gaps of the power transformer magnetic cores. In order to numerically predict the effectiveness of the proposed method, a 3-D magneto-mechanical strong coupled model including MS and magnetic anisotropy of steel sheet was founded. Then, the numerical model was applied to analyze the step-lap joint region of the corner of magnetic cores. The analysis results illustrated that the deformation and noise of core with NSMC are lower than with the traditional epoxy damping material. Moreover, the validity of the proposed new way was verified by the simplified step-lap joint cores, which were achieved based on Epstein Frames. @ 2013 AIP Publishing LLC. [http://dx.doi.org/10.1063/1.4800077]
\end{abstract}

\section{INTRODUCTION}

To reduce the vibration and noise of power transformer cores, Phway et al. avoided resonance between the natural frequencies of the structure and the driving frequency ${ }^{1}$ and Yanhui et al. proposed a method by inserting the gap in the yoke and choosing the hard materials for the gaps. ${ }^{2}$ But it is difficult to fix the yoke with gaps by the present manufacturing method. To further reduce the electromagnetic noise of transformer cores, nanocrystalline soft magnetic composite (NSMC) material, which is the composition of nano-sized magnetic material with high permeability and rubber particles, instead of epoxy or asphalt traditional damping material ${ }^{3}$ is first introduced to fill the multi-joint gaps of the cores. When the joint gaps are filled by NSMC, the magnetic flux density and magnetic flux leakage will be reduced in the thickness direction, so the magnetic forces (MF) and magnetostriction (MS) off the laminated plane will also be lessened. In addition, NSMC plays damping as epoxy for its rubber particles. Therefore, the proposed method can reduce the vibration and noise of cores effectively.

\section{FINITE ELEMENT ANALYSIS}

\section{A. Magneto-elastic coupled model}

A full 3-D numerical modeling of a power transformer core is computationally demanding; so, for analysis, the overlap

\footnotetext{
${ }^{\text {a) }}$ Author to whom correspondence should be addressed. Electronic mail: yanrg@hebut.edu.cn.
}

region with multistep lap (MSL), which has a key impact on core vibration and losses, is built and shown in Fig. 1.

Based on our previous researches, ${ }^{4,5}$ the total energy functional of the transformer cores includes mechanical energy, magnetic energy, and magnet-mechanical coupling energy, which can be expressed as follows:

$$
\begin{aligned}
I= & \int_{\Omega_{2}}\left(\frac{1}{2} \sigma^{T} \boldsymbol{s}^{H} \sigma\right) d V+\int_{\Omega_{2}}\left(\sigma^{T} \boldsymbol{d} \boldsymbol{H}\right) d V+\int_{\Omega_{1}}\left(\frac{1}{2} \boldsymbol{H}^{T} \boldsymbol{\mu}^{\sigma} \boldsymbol{H}\right) d V \\
& -\int_{\Omega_{1}} \boldsymbol{J} \cdot \boldsymbol{A} d V-\int_{\Gamma_{1}} \boldsymbol{f}_{\Gamma} \cdot \boldsymbol{u} d V-\int_{\Omega_{2}} \boldsymbol{f}_{V} \cdot \boldsymbol{u} d V
\end{aligned}
$$

where $\boldsymbol{A}$ is the magnetic vector potential and $\boldsymbol{B}=\nabla \times \boldsymbol{A}, \boldsymbol{u}$, the mechanical displacement, $\boldsymbol{\varepsilon}$ and $\boldsymbol{\sigma}$, the vector of strain and stress, and $\boldsymbol{d}$, the MS coefficient matrix.

Coefficients $d_{11}$ and $d_{22}$ can be obtained from measured MS characteristic curves $\lambda_{x}\left(B_{x}\right)$ and $\lambda_{y}\left(B_{y}\right){ }^{4}$ If shearing strains of the steel lamination are neglected, there is $d_{i j}=0(i=4,5$, 6 and $j=1,2,3)$. The MS coefficient in the normal direction is assumed as $d_{33}=\left(d_{11}+d_{22}\right) / 2$. Using the Hooker's law, we can get $d_{21}=d_{31}=-\alpha d_{11}, \quad d_{12}=d_{32}=-\alpha d_{22}$, and $d_{13}=d_{23}=-\alpha d_{33}$, where $\alpha$ is the Poisson ratio. So, the magneto-mechanical coupling energy is given by

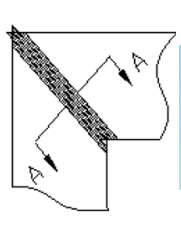

$$
\underline{\mathrm{A}-\mathrm{A}}
$$

Air Gap
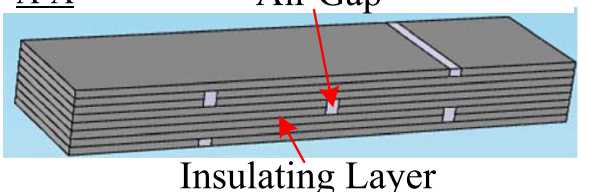

Insulating Layer

FIG. 1. MSL assembling in joint region (illustrated for $N=3$ ). 


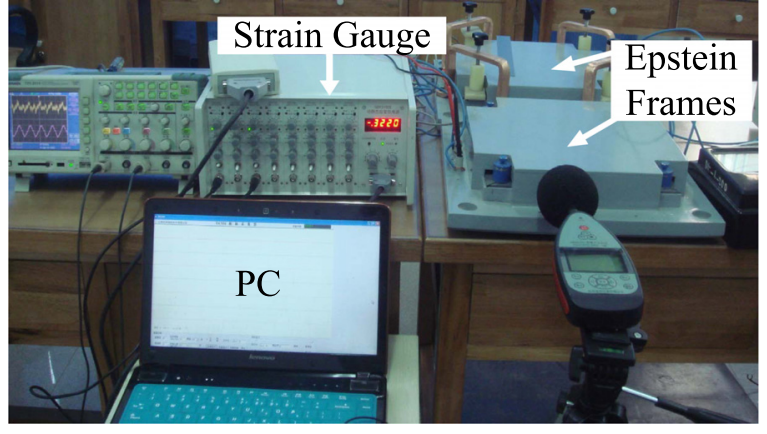

FIG. 2. The vibration and noise measurement system.

$$
\begin{aligned}
\int_{\Omega_{2}} \sigma^{T} d H d V= & E \int_{\Omega_{2}}\left(d_{11} v_{x} B_{x} \varepsilon_{x}+d_{22} v_{y} B_{y} \varepsilon_{y}\right. \\
& \left.+d_{33} v_{z} B_{z} \varepsilon_{z}\right) d x d y d z,
\end{aligned}
$$

where $E$ is the Young's modulus.

After element discretization of functional $I$ and element assembly, ${ }^{5}$ matrix equation of the magneto-mechanical system is given by

$$
\left(\begin{array}{ll}
M & D \\
C & K
\end{array}\right)\left(\begin{array}{l}
A \\
u
\end{array}\right)=\left(\begin{array}{c}
J \\
f_{v}+f_{\Gamma}
\end{array}\right)
$$

where $\boldsymbol{M}$ is the electromagnetic matrix, $\boldsymbol{K}$, the mechanical stiffness matrix, $\boldsymbol{C}, \boldsymbol{D}$, the coupling interactions between the magnetic field and mechanical deformation, and $\boldsymbol{C}=\boldsymbol{D}^{T}$.

\section{B. Acoustical analysis}

Based on the vibration calculation, according to classical theory, ${ }^{6}$ the sound power radiated by the core can be expressed as

$$
\begin{aligned}
W & =\rho c k_{i} \oint_{S} v_{n}^{2} d S_{c}=\rho_{0} c_{0} k_{i} \sum_{i} v_{n, j}^{2} S_{c, j} \\
& =\rho_{0} c_{0} k_{i} \sum_{i}\left(\frac{\partial u_{n, j}}{\partial t}\right)^{2} S_{c, j},
\end{aligned}
$$

where $\rho c$ is the characteristic impedance of the noise transmission medium and $k_{i}$ the radiation coefficient of the $i$ core surface.

Then, we can get the sound pressure level $L_{p}$ of the free sound field around the core

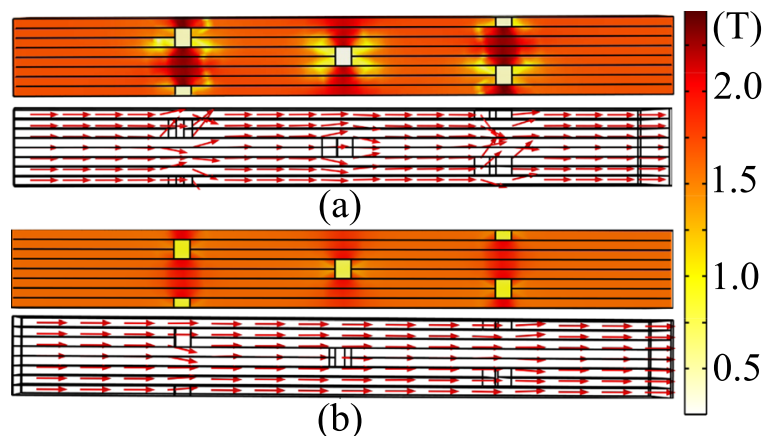

FIG. 3. Magnetic flux density distribution in the joint region: (a) gaps with epoxy; (b) gaps with NSMC.

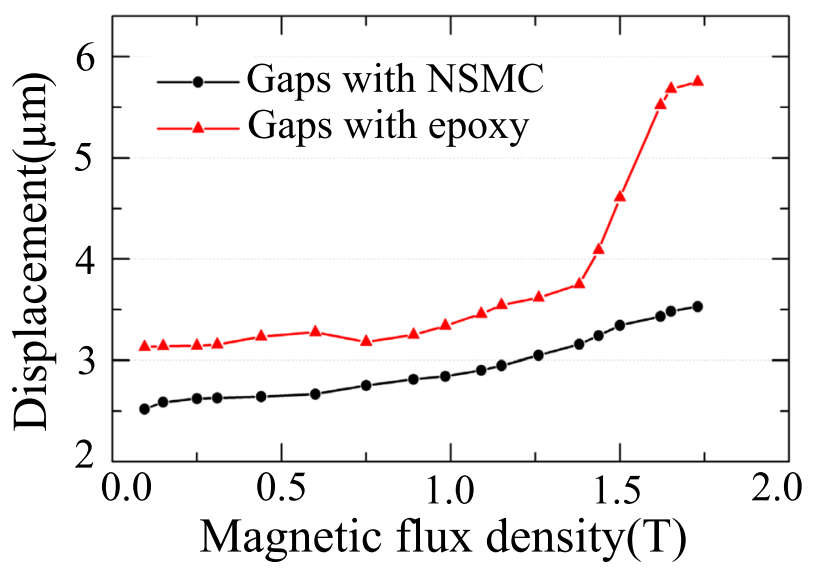

FIG. 4. Stress on the deformed step-lap joint due to MS and MF: (a) gaps with epoxy; (b) gaps with NSMC.

$$
L_{p}=10 \lg \left(W / 10^{-12}\right)-20 \lg R-11,
$$

$W$ is the power calculated by Eq. (4) and $R$ the distance from the measured point to the core.

\section{EXPERIMENTS}

The vibration and noise measurement system is shown in Fig. 2. For comparison tests, two cores with step-lap joint were assembled based on $25 \mathrm{~cm}$ standard Epstein Frames. The steels of 30Q120 $30 \mathrm{~mm}$ in width and $285 \mathrm{~mm}$ in length were cut along $45^{\circ}$ at both ends and stacked with 3 steps. Similar to analysis, one core's joint gaps were filled with NSMC and the other with epoxy.

Dynamic strain gauge and foil-type resistance strain gauges were used to measure the deformation of the core and the noise level was recorded by noise analyzer AWA6270+e. Both vibration and noise measurements were completed in a mute laboratory.

\section{RESULTS AND DISCUSSION}

\section{A. Analysis results}

Based on the proposed strong coupled model, using pseudo-source technique, ${ }^{7}$ the magnetic flux density in the joint region is calculated and shown in Fig. 3. It is obviously that the interlaminar flux $B_{z}$ is exhibited at the overlapped gaps, which yields a compressive off-plane strain and the magnetic flux lines in the condition of the gap filled with epoxy bend much more than with NSMC. This is because

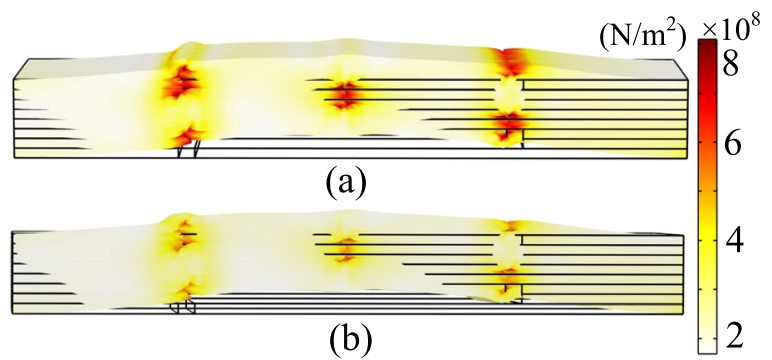

FIG. 5. Noise level around the 1/4 step-lap core: (a) gaps with epoxy; (b) gaps with NSMC. 


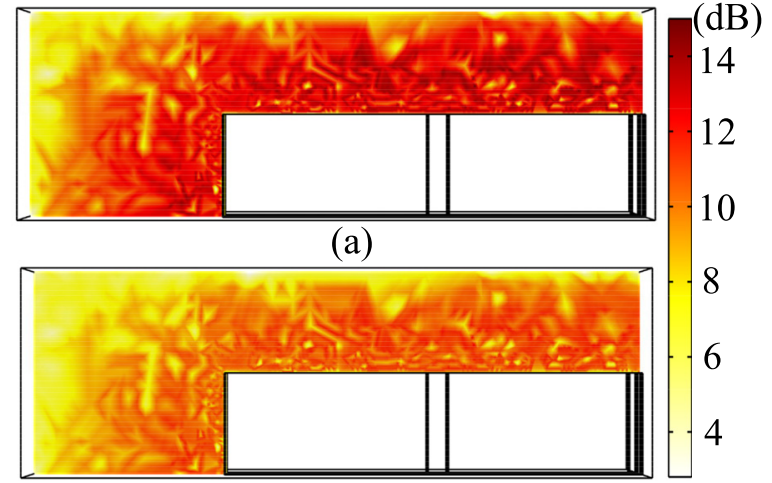

(b)

FIG. 6. The measurement results of the vibration of the cores with different damping materials.

NSMC not only has damping effect but also has magnetic characteristic that helps the magnetic flux through the lap gaps. Once NSMC is filled in the joint gaps, the interlaminar flux $B_{z}$, which induces off-plane MS stress and MF, will be lessened. As illustrated in Fig. 4, the stress at the ends of the sheets of the core with epoxy is up to $8 \times 10^{8} \mathrm{~N} / \mathrm{m}^{2}$, while the core with NSMC is $4.5 \times 10^{8} \mathrm{~N} / \mathrm{m}^{2}$. According to Eq. (5), the result of noise level around the cores is shown in Fig. 5 , in which the noise level of the core with epoxy is larger than the model with NSMC. It is revealed that the level around the core with NSMC is lower about $3 \mathrm{~dB}$ than with epoxy at the same distance from the core.

\section{B. Experimental results}

According to the strain measurement results, the deformation of a local position close to the joint gap of each core is shown in Fig. 6. Obviously, the overall displacement of the core with NSMC is lower than with epoxy and NSMC has a more pronounced reduction effect when the magnetic flux density is greater than $1.5 \mathrm{~T}$. The large deformation, $5.75 \mu \mathrm{m}$, can be reduced by the core with epoxy, while the core with NSMC is only $3.53 \mu \mathrm{m}$ correspondingly when the magnetic density is $1.73 \mathrm{~T}$. The MF and MS in $z$ direction at the joint gaps are much smaller because magnetic flux would be smoother by NSMC, which is similar to the numerical analysis results.

The noise level of each model obtained by measurement is listed in Table I. Noise level of $41.6 \mathrm{~dB}$ is generated by the core with epoxy, while the noise level can be reduced to $35.6 \mathrm{~dB}$ with NSMC when magnetic density is $1.73 \mathrm{~T}$ and the proposed method can reduce noise by an average of $5.9 \mathrm{~dB}$ when magnetic flux density is greater than $1.5 \mathrm{~T}$.

In this experiment, we can verify that the method can reduce the vibration and noise of core and it is in accordance with the analyzed results. However, the effect of the
TABLE I. Measured noises of the two cores (the background noise level is $19.0 \mathrm{~dB})$.

\begin{tabular}{lcc}
\hline \hline & \multicolumn{2}{c}{ Noise level (dB) } \\
\cline { 2 - 3 } Magnetic flux density (T) & Gaps with epoxy & Gaps with NSMC \\
\hline 0.3 & 22.3 & 21.9 \\
0.6 & 23.5 & 23.1 \\
0.9 & 24.8 & 24.5 \\
1.4 & 34.8 & 29 \\
1.5 & 38 & 31.5 \\
1.6 & 38.7 & 32.2 \\
1.65 & 39.6 & 34.3 \\
1.7 & 41.1 & 35.2 \\
1.73 & 41.9 & 35.6 \\
\hline \hline
\end{tabular}

proposed way applied to power transformer needs further validation, for Epstein frame core is simplified and singlephase.

\section{CONCLUSION}

Vibration and noise of transformer cores can be reduced by using NSMC material to fill the step-lap joints gap. In order to test the new method, a magneto-mechanical strong coupled model for laminated cores including MS was founded and based on which the vibration deformation and noise level of MSL cores with NSMC and epoxy were comparatively analyzed. Computation results declare that the proposed method has better noise reduction than traditional methods. The effectiveness of the new method is also verified by experimental study of Epstein Frame cores.

\section{ACKNOWLEDGMENTS}

The research work was supported by National Natural Science Foundation of China (51237005, 51177038, 51107027) and Natural Science Foundation of Tianjin (12JCDJ286000) and Hebei Province (E2012202103).

${ }^{1}$ T. P. P. Phway and A. J. Moses, J. Magn. Magn. Mater. 316(2), 468 (2007). ${ }^{2}$ Y. Gao, K. Muranatsu, and H. Muhd Juzail, IEEE Trans. Magn. 46(6), 2179 (2010).

${ }^{3}$ S. H. Ravi, K. P. Vamsi, R. V. Bhujanga, and B. P. Bangaru, Proceedings of IMechE, Part L: Journal of Materials: Design and Appl. 224(2), 63 (2010).

${ }^{4}$ Z. Lihua, Y. Qingxin, and Y. Rongge, in Proceedings of the 6th ICEF (2012), p. D333.

${ }^{5}$ Q. Yang, R. Yan, C. Fan, and H. Chen, IEEE Trans. Magn. 43(4), 1437 (2007).

${ }^{6}$ L. Taibao, Computational Acoustics-Equations and Calculation Methods of Sound Field [M] (Science Press, 2005).

${ }^{7}$ T. D. Kefalas, G. Loizos, and A. G. Kladas, IEEE Trans. Magn. 47(5), 1058 (2011). 